\title{
Desnutrição e sua associação com atividades durante o lazer entre adolescentes
}

\author{
Malnutrition and its association with activities \\ during leisure time among adolescents
}

Rômulo Araújo Fernandes ${ }^{1}$

Diego Giulliano Destro Christofaro ${ }^{1}$

Raphael Mendes Ritti Dias ${ }^{2}$

Jamile Sanches Codogno ${ }^{1}$

Arli Ramos de Oliveira ${ }^{3}$

${ }^{1}$ Laboratório de Investigação em Exercício (LIVE).

Departamento de Educação Física, Faculdade de Ciências e Tecnologia de Presidente Prudente, Universidade Estadual Paulista Júlio de Mesquita Filho. R. Roberto Simonsen

305/Departamento de Educação Física (Sala 97), Centro Educacional. 19.060-900 Presidente Prudente SP Brasil. romulo_ef@yahoo.com.br

${ }^{2}$ Escola Superior de Educação Física,

Universidade de Pernambuco.

${ }^{3}$ Departamento de Ciências do Esporte, Centro de

Educação Física e Esporte, Universidade Estadual de

Londrina.
Abstract The scope of this study is to analyze the prevalence of malnutrition among adolescents in two Brazilian cities and to analyze possible associations with leisure-time physical activity. 2782 adolescents ranging from 10 to 17 years of age enrolled in public and private schools from a city in the southeastern and another in the southern region participated in the study. Body weight and height were duly recorded. Physical activity was assessed: sports, walking; cycling and watching television. The Chi-square and Poisson regression tests were applied. The prevalence of malnutrition was $13 \%$, being higher among females (14.5\%) than among males (11.9\%) ( $p=0.047)$. Practicing sport was associated with a lower occurrence of the condition ( $p=0.001$ ). The prevalence of malnutrition was significantly lower among those who maintained moderate walking frequency in comparison with those who walked less. The overall prevalence of malnutrition in this study was considered to be high. In addition to this, inadequate nutritional status in adolescents seems associated with risk-related health behavior, such as insufficient leisure-time physical activity.

Key words Malnutrition, Physical activity, Adolescents
Resumo O objetivo deste artigo é verificar a prevalência de desnutrição em adolescentes de duas cidades brasileiras e analisar possíveis associações com a prática de atividades físicas durante horários de lazer. Participaram do estudo 2782 adolescentes de 10 a 17 anos, de escolas da rede pública e privada de uma cidade do sudeste e uma da região sul. O peso e a estatura foram avaliados por balança digital e estadiômetro, respectivamente. A prática de atividade física considerou as variáveis: prática de esportes; andar (tratada como caminhada); andar de bicicleta e assistir televisão. $O$ teste qui-quadrado e a regressão de Poisson foram empregados. A prevalência de desnutrição foi de $13,0 \%$, sendo maior no sexo feminino $(14,5 \%)$ quando comparado ao masculino $(11,9 \%)[p=$ $0,047]$. A prática esportiva foi associada com menor ocorrência do desfecho $(p=0,001)$. Foi significativamente menor a prevalência de desnutrição entre aqueles que mantinham frequência moderada de caminhadas em comparação àqueles que apresentavam baixa frequência dessa prática. A prevalência de desnutrição no presente estudo pode ser considerada elevada. Além disso, a condição nutricional inadequada nos adolescentes aparentemente está associada a comportamentos de risco à saúde, caso da prática insuficiente de atividade física durante horários de lazer.

Palavras-chave Desnutrição, Atividade física, Adolescentes 


\section{Introdução}

A desnutrição na infância e adolescência é um dos problemas de saúde pública da atualidade. No ano de 1997 , aproximadamente $8,6 \%$ das crianças e adolescentes apresentavam tal desfecho ${ }^{1}$, sendo uma das principais causas de morte nessas fases de vida nos países em desenvolvimento. Além do aumento de risco de mortalidade, a desnutrição crônica na infância está associada com a ocorrência de doenças crônicas não transmissíveis que podem trazer repercussão na idade adulta. Tem sido demonstrado que crianças e adolescentes desnutridos apresentam maior prevalência de hipertensão arterial $^{1}$, bem como atraso no crescimento e diminuição do desempenho cognitivo ${ }^{2}$.

A prática de atividade física tem sido recomendada como importante estratégia de saúde pública para melhoria da saúde de crianças e adolescentes. Adolescentes fisicamente ativos apresentam menor morbidade e agravos à saúde na idade adulta ${ }^{3}$, além da proteção de fatores de risco cardiovasculares na infância e adolescência $^{4}$. Apesar dessas evidências, poucos estudos foram conduzidos com o objetivo de analisar o nível de atividade física de adolescentes com desnutrição. Um estudo recente evidenciou que adolescentes desnutridos apresentam menores níveis de atividade física comparados aos eutróficos ${ }^{5}$. Todavia, como este estudo analisou a atividade física geral, pouco se sabe sobre outros comportamentos de risco como o sendentarismo e o impacto da desnutrição em tipos específicos de atividade física, como por exemplo, a atividade física de lazer.

Assim, o objetivo do presente estudo foi analisar a prevalência de desnutrição em adolescentes de duas cidades brasileiras e sua associação com a prática de atividades físicas durante horários de lazer.

\section{Material e métodos}

A amostra do presente estudo foi composta por 2782 adolescentes de 10 a 17 anos matriculados em escolas públicas e privadas das cidades de Presidente Prudente (PP) (maior cidade da região oeste do Estado de São Paulo) e Londrina (LO) (maior cidade da região norte do Estado do Paraná), cidades estas distantes não mais do que $200 \mathrm{~km}$.

Em ambos os levantamentos, o estado nutricional foi o desfecho principal utilizado para o calculo amostral. Informações mais detalhadas sobre os processos de seleção da amostra são apresentados em publicações anteriores ${ }^{6,7}$. Em PP, 1779 adolescentes foram avaliados, porém, para este estudo, 18 foram excluídos devido a problemas no preenchimento sobre comportamentos relacionados à prática de atividades físicas durante horários de lazer, totalizado, assim, 1761 adolescentes. Em LO, foram avaliados 1144 adolescentes, sendo excluídos 129 por preenchimentos inconsistentes dos questionários, totalizando 1015 para este estudo.

Em ambas as cidades, a coleta de dados foi conduzida seguindo a mesma metodologia. Os pesquisadores responsáveis pelos dois estudos participaram das coletas em ambas as cidades. Os levantamentos foram conduzidos em épocas diferentes (segundo semestre do ano de $2007 \mathrm{em}$ Presidente Prudente; primeiro semestre do ano de 2008 em Londrina). O estudo foi aprovado pelo comitê de ética e pesquisa das instituições envolvidas na pesquisa.

A desnutrição foi avaliada por meio dos valores de massa corporal (em quilogramas $[\mathrm{kg}]$ ) e a estatura (em metros $[\mathrm{m}]$ ) que foram aferidos com a utilização de balança eletrônica e estadiômetro, respectivamente. Durante as medidas os avaliados permaneceram descalços e trajados com roupas leves. O índice de massa corporal (IMC) foi calculado com os valores de massa corporal e estatura (expresso em $\mathrm{kg} / \mathrm{m}^{2}$ ), e para o diagnóstico da presença da desnutrição foram utilizados os valores críticos ajustados por sexo e idade propostos por Cole et al. ${ }^{8}$. Todas as medidas antropométricas foram realizadas dentro da própria unidade escolar por pesquisadores previamente treinados e experientes.

Para avaliação do nível de atividade física no lazer foi utilizado o questionário proposto por Baecke et al. ${ }^{9}$. No presente instrumentos foram consideradas as variáveis: (i) prática de esportes; (ii) andar (tratada como caminhada a partir deste momento); (iii) andar de bicicleta (tratada como ciclismo a partir deste momento); (iv) assistir televisão. A prática de esportes foi tratada como variável dicotômica (sim ou não) para o envolvimento atual em alguma atividade esportiva durante horários de lazer. As demais variáveis, por terem cinco possíveis respostas (nunca; raramente; algumas vezes; frequentemente; sempre), foram agrupadas em apenas três grupos (Baixa frequência [Baixa-F]: categorias "nunca" e "raramente"; Moderada frequência [Moderada-F]: categorias "algumas vezes" e "frequentemente"; Alta frequência [Alta-F]: categoria "sempre") seguindo metodologia previamente publicada ${ }^{6}$. 
Em PP, alguns adolescentes não reportaram informações referentes a apenas um dos quatro comportamentos durante horários de lazer e foram mantidos nas análises. Assim, as análises para caminhada, ciclismo e televisão foram conduzidas com 1739, 1747 e 1750, excluídos, respectivamente, 22, 14 e 11 adolescentes. Em LO, pelo mesmo motivo, foram excluídos seis adolescentes para essas mesmas variáveis, totalizando 1015. Análises adicionais com a exclusão destes indivíduos não modificaram os dados aqui apresentados.

O teste qui-quadrado foi empregado para comparar proporções e analisar associações. Quando a tabela de contingência criada era de formação " 2 x2" a correção de Yates foi empregada, nos demais casos empregou-se um teste de associação linear. Para analisar a magnitude das associações foi empregada a regressão de Poisson com ajuste robusto de variância (expressa como razão de prevalência $[\mathrm{RP}]$ e seu respectivo intervalo de confiança de 95\% [IC95\%]). A opção pela regressão de Poisson ao invés da logística binária foi pautada no fato da primeira, munida do ajuste robusto de variância, ser uma medida mais conservadora para a análise das magnitudes observadas. Além disso, o desfecho principal analisado mostrou-se superior a $10 \%$, prejudicando assim a perspectiva de se utilizar medidas de razão de chance. Por fim, criou-se um modelo ajustado para a análise multivariada, no qual as possíveis variáveis de confusão: (i) sexo, (ii) idade e (iii) cidade, foram inseridas, inicialmente uma de cada vez e, finalmente, fez-se a entrada de todas simultaneamente.

Previamente a todas as análises, para se considerar o resultado significativo do ponto de vista estatístico, utilizou-se um valor de significância $(p)$ inferior a 5\%. O software estatístico STATA em sua versão 8.0 gerou todas as análises apresentadas.

\section{Resultados}

Aproximadamente 53\% $(\mathrm{n}=1472)$ dos adolescentes incluídos eram do sexo feminino e esta proporção não diferiu significativamente entre as duas cidades $(p=0,355)$. Os adolescentes de LO apresentaram menor prática de ciclismo ( $p=$ 0,012), ao passo que os adolescentes de PP apresentaram maior prática de atividades sedentárias durante horários de lazer, caso da televisão ( $p$ $=0,001)$. A prática esportiva $(p=0,742)$ e de caminhada $(p=0,479)$ foram similares nas duas cidades (Tabela 1 ).
A prevalência de desnutrição foi de 13,3\% (IC95\% = 12\% - 14,6\%), sem diferenças entre as cidades $(p=0,141)$ e grupos etários $(p=0,291)$. A presença da desnutrição foi maior no sexo feminino (Feminino: 14,5\% e Masculino: 11,9\%; $p$ $=0,047$ ).

No que se refere aos comportamentos durante o lazer (Tabela 2), a prática esportiva foi associada com menor ocorrência do desfecho ( $p$ $=0,001)$. Por outro lado, o mesmo padrão não foi observado para as variáveis: caminhada ( $p=$ $0,084)$, ciclismo $(p=0,358)$ e assistir televisão $(p$ $=0,893$ ).

Em todos os modelos testados (Tabela 3), a prática esportiva foi significativamente inferior entre adolescentes desnutridos ( RP $=0.64$ [IC95\% $=0.52-0.79])$. Além disso, a desnutrição também se associou com menor ocorrência de caminhada durante horários de lazer. Foi significativamente menor a prevalência de desnutrição entre aqueles que mantinham frequência moderada de caminhadas em comparação àqueles que apresentavam baixa frequência dessa prática (RP $=0.79[$ IC95\% $=0.63-0.98])$.

\section{Discussão}

Estudo transversal realizado em duas cidades brasileiras que evidenciou elevada prevalência de desnutrição em adolescentes, sendo que esta condição esteve associada com a atividade física habitual.

A prevalência de desnutrição encontrado no presente estudo foi de 13,3\%, valores similares aos observados por Wang et al. ${ }^{1}$ na década de 1970 no Brasil, onde especificamente nas regiões sudeste e nordeste a prevalência encontrada foi de 14,8\%. Embora Monteiro et al. ${ }^{10}$ tenham observado declínio na prevalência de desnutrição entre crianças brasileiras com idade inferior a cinco anos no período de 1996 e 2006/2007, de 13,5\% para $6,8 \%$; os dados apresentados para adolescentes não apresentam a mesma tendência.

Em nosso estudo, o sexo feminino foi associado à maior prevalência de desnutrição. Embora os motivos envolvidos na maior prevalência de desnutrição nas adolescentes do sexo feminino não tenham sido analisados neste estudo, sabese que a adolescência é um período de consolidação da personalidade do futuro adulto, na qual ele busca sua aceitação em algum grupo específico e, muitas vezes, o jovem julga que assumir o padrão estético ideal vigente proporcione maior aceitação, ou constitua fator determinante para 
Tabela 1. Características gerais de adolescentes de acordo com o município analisado.

\begin{tabular}{|c|c|c|c|c|}
\hline Variáveis & $\begin{array}{c}\text { Total }(\mathrm{n}=2782) \\
\mathrm{N}(\%)\end{array}$ & $\begin{array}{l}\text { Pres. Prudente }(\mathbf{n}=1761) \\
\text { N }(\%)\end{array}$ & $\begin{array}{c}\text { Londrina }(\mathbf{n}=1021) \\
\text { N }(\%)\end{array}$ & $\mathrm{p}$ \\
\hline \multicolumn{5}{|l|}{ Desnutrição } \\
\hline Sim & $370(13,3)$ & $221(12,5)$ & $149(14,6)$ & \multirow[t]{2}{*}{0,141} \\
\hline Não & $2412(86,7)$ & $1540(87,5)$ & $872(85,4)$ & \\
\hline \multicolumn{5}{|l|}{ Sexo } \\
\hline Masculino & $1310(47,1)$ & $817(46,4)$ & $493(48,3)$ & \multirow[t]{2}{*}{0,355} \\
\hline Feminino & $1472(52,9)$ & $944(53,6)$ & $528(51,7)$ & \\
\hline \multicolumn{5}{|l|}{ Idade } \\
\hline 10 a 12 anos & $1144(41,1)$ & $510(29,0)$ & $634(62,1)$ & \multirow[t]{3}{*}{$0,001^{*}$} \\
\hline 13 a 14 anos & $924(33,2)$ & $593(33,7)$ & $331(32,4)$ & \\
\hline 15 a 17 anos & $714(25,7)$ & $658(37,3)$ & $56(5,5)$ & \\
\hline \multicolumn{5}{|l|}{ Esporte } \\
\hline Sim & $1726(62,0)$ & $1088(61,8)$ & $638(62,5)$ & \multirow[t]{2}{*}{0,742} \\
\hline Não & $1056(38,0)$ & $673(38,2)$ & $383(37,5)$ & \\
\hline \multicolumn{5}{|l|}{ Caminhada } \\
\hline Baixa-F & $1329(48,3)$ & $832(47,8)$ & $497(49)$ & \multirow[t]{3}{*}{$0,479^{*}$} \\
\hline Moderada-F & $1177(42,7)$ & $746(42,9)$ & $431(42,5)$ & \\
\hline Alta-F & $248(9,0)$ & $161(9,3)$ & $87(8,6)$ & \\
\hline \multicolumn{5}{|l|}{ Ciclismo } \\
\hline Baixa-F & $1297(47)$ & $802(45,9)$ & $495(48,8)$ & \multirow[t]{3}{*}{$0,012^{*}$} \\
\hline Moderada-F & $1042(37,7)$ & $650(37,2)$ & $392(38,6)$ & \\
\hline Alta-F & $423(15,3)$ & $295(16,9)$ & $128(12,6)$ & \\
\hline \multicolumn{5}{|l|}{ Televisão } \\
\hline Baixa-F & $344(12,4)$ & $150(8,6)$ & $194(19,1)$ & \multirow[t]{3}{*}{$0,001^{*}$} \\
\hline Moderada-F & $1531(55,4)$ & $955(54,6)$ & $576(56,7)$ & \\
\hline Alta-F & $890(32,2)$ & $645(36,9)$ & $245(24,1)$ & \\
\hline
\end{tabular}

" = teste qui-quadrado para associação linear. Baixa-F = frequência baixa; Moderada-F = frequência moderada; Alta- $\mathrm{F}=$ frequência alta.

Tabela 2. Associação univariada entre desnutrição e indicadores de atividade física durante horários de lazer entre adolescentes de dois municípios brasileiros.

\begin{tabular}{lcc}
\hline $\begin{array}{c}\text { Atividades em } \\
\text { horários de lazer }\end{array}$ & $\begin{array}{c}\text { Desnutrição } \\
\text { N }(\%)\end{array}$ & $\begin{array}{c}\text { Qui-quadrado } \\
\boldsymbol{p}\end{array}$ \\
\hline Esporte & & 0,001 \\
$\quad$ Sim & $188(10,9)$ & \\
Não & $182(17,2)$ & \\
Caminhada & & 0,084 \\
$\quad$ Baixa-F & $196(14,7)$ & \\
Moderada-F & $139(11,8)$ & \\
Alta-F & $32(12,9)$ & \\
Ciclismo & & 0,358 \\
Baixa-F & $172(13,3)$ & \\
Moderada-F & $151(14,5)$ & \\
Alta-F & $44(10,4)$ & \\
Televisão & & \\
Baixa-F & $41(11,9)$ & \\
Moderada-F & $211(13,8)$ & \\
Alta-F & $115(12,9)$ & \\
\end{tabular}

Baixa- $\mathrm{F}$ = frequência baixa; Moderada- $\mathrm{F}$ = frequência moderada; Alta- $\mathrm{F}=$ frequência alta. tal aceitação ${ }^{11}$. Similarmente, Branco et al. ${ }^{12}$, verificaram que adolescentes do sexo feminino de 14 a 19 anos residentes na cidade de São Paulo apresentaram maior preocupação com sua imagem corporal, fenômeno não observado entre jovens do sexo masculino. É importante salientar também que a preocupação excessiva em emagrecer pode gerar uma alteração de comportamento alimentar que leva ao surgimento de outras doenças como, por exemplo. a anorexia nervosa, que geralmente ocorre na adolescência e cerca de $90 \%$ dos atingidos pela doença são do sexo feminino ${ }^{13,14}$.

A desnutrição é uma doença multifatorial e seu diagnóstico é mais provável em adolescentes com baixa estatura, com atraso na maturação puberal, com alimentação deficiente e com problemas sociais, como falta de vinculo familiar ${ }^{15}$, da mesma forma o nível de atividade física pode influenciar o surgimento da doença. Uma variedade de comportamentos sedentários durante horários de lazer foi associada com a presença da desnutrição no presente estudo. Pinheiro et 
Tabela 3. Associação multivariada entre desnutrição e indicadores de atividade física durante horários de lazer entre adolescentes de dois municípios brasileiros.

\begin{tabular}{|c|c|c|c|c|}
\hline $\begin{array}{c}\text { Atividades em } \\
\text { horários de lazer }\end{array}$ & $\begin{array}{c}\text { Modelo 1 } \\
\text { RP (IC95\%) }\end{array}$ & $\begin{array}{c}\text { Modelo } 2 \\
\text { RP (IC95\%) }\end{array}$ & $\begin{array}{c}\text { Modelo } 3 \\
\text { RP (IC95\%) }\end{array}$ & $\begin{array}{c}\text { Modelo } 4 \\
\text { RP (IC95\%) }\end{array}$ \\
\hline \multicolumn{5}{|l|}{ Esporte } \\
\hline Sim & $0.64(0.52-0.79)$ & $0.63(0.51-0.77)$ & $0.63(0.51-0.77)$ & $0.64(0.52-0.79)$ \\
\hline Não & $1.00(-)$ & $1.00(-)$ & $1.00(-)$ & $1.00(-)$ \\
\hline \multicolumn{5}{|l|}{ Caminhada } \\
\hline Baixa-F & $1.00(-)$ & $1.00(-)$ & $1.00(-)$ & $1.00(-)$ \\
\hline Moderada-F & $0.79(0.63-0.98)$ & $0.79(0.64-0.99)$ & $0.80(0.64-0.99)$ & $0.79(0.63-0.98)$ \\
\hline Alta-F & $0.86(0.59-1.26)$ & $0.87(0.60-1.27)$ & $0.87(0.60-1.27)$ & $0.87(0.60-1.27)$ \\
\hline \multicolumn{5}{|l|}{ Ciclismo } \\
\hline Baixa-F & $1.00(-)$ & $1.00(-)$ & $1.00(-)$ & $1.00(-)$ \\
\hline Moderada-F & $1.12(0.90-1.40)$ & $1.11(0.89-1.38)$ & $1.09(0.87-1.36)$ & $1.16(0.93-1.46)$ \\
\hline Alta-F & $0.83(0.59-1.17)$ & $0.80(0.57-1.11)$ & $0.79(0.56-1.10)$ & $0.89(0.63-1.26)$ \\
\hline \multicolumn{5}{|l|}{ Televisão } \\
\hline Baixa-F & $1.00(-)$ & $1.00(-)$ & $1.00(-)$ & $1.00(-)$ \\
\hline Moderada-F & $1.14(0.81-1.59)$ & $1.15(0.82-1.61)$ & $1.19(0.85-1.67)$ & $1.19(0.84-1.66)$ \\
\hline Alta-F & $1.04(0.72-1.49)$ & $1.09(0.76-1.56)$ & $1.13(0.79-1.63)$ & $1.14(0.79-1.65)$ \\
\hline
\end{tabular}

$\mathrm{RP}=$ razão de prevalência; IC95\% = intervalo de confiança de 95\%; Baixa-F = frequência baixa; Moderada-F = frequência moderada; Alta-F = frequência alta; Modelo $1=$ ajustado por sexo; Modelo $2=$ ajustado por idade; Modelo $3=$ ajustado por Cidade; Modelo 4 = ajustado por sexo, idade e cidade.

al. ${ }^{16}$ identificaram, entre adolescentes da região sul do país, que o sedentarismo, bem como a insatisfação com a imagem corporal, foram associados com a ocorrência de transtornos mentais comuns (depressão, ansiedade, etc.), os quais podem contribuir para a manutenção de comportamentos sedentários. Em nosso estudo, possivelmente, a associação entre maior prevalência de desnutrição, prática de atividades físicas e atividades sedentárias pode ser atribuída a uma causalidade reversa, uma vez que jovens com alguma insatisfação corporal têm menor probabilidade de se engajar em atividades que envolvam alguma possível exposição de sua forma física indesejada ${ }^{17}$.

A utilização de pontos de corte mais recentes para identificar desnutrição ${ }^{8}$ limita comparações com estudos previamente realizados e precisa ser apontada como limitação. Porém, a principal limitação do presente estudo reside em seu delineamento transversal, que não permite estabelecer relações de causalidade entre a presença da desnutrição e os comportamentos durante horários de lazer analisados. Da mesma forma, os indícios encontrados na literatura ${ }^{12,16}$ nos permitem construir modelos teóricos para identificar qual é a direção desta associação, porém, identificam de maneira clara o grande impacto que a causa- lidade reversa pode ter sobre a interpretação desta associação. Assim, faz-se necessária a elaboração de projetos de delineamento transversal abordando o assunto.

Apesar dos achados do presente estudo, ressalta-se que muita ênfase vem sendo dada ao aumento do sobrepeso/obesidade ${ }^{18-22}$, porém estudos com o objetivo de avaliar a prevalência de desnutrição e possíveis fatores de risco associados são poucos, mesmo a prevalência de desnutrição ainda sendo considerada alta, mesmo em regiões consideradas mais desenvolvidas, como é o caso do presente estudo.

\section{Conclusões}

Em resumo, é possível concluir que, embora analisando adolescentes de duas cidades brasileiras provenientes de regiões desenvolvidas do país, foi possível identificar uma elevada prevalência de desnutrição. Além disso, esta condição nutricional aparentemente está associada a uma variedade de comportamentos de risco à saúde, caso da inatividade física durante horários de lazer. Assim, futuras investigações devem se aprofundar mais nas causas deste evento, bem como a efetividade de possíveis ações visando o seu combate. 


\section{Colaboradores}

RA Fernandes, DGD Christofaro, RMR Dias, JS Codogno e AR Oliveira participaram de todas as etapas de elaboração do artigo, desde o delineamento do estudo, a coleta e análise das informações até a redação final.

\section{Referências}

1. Wang Y, Monteiro C, Popkin BM. Trends of obesity and underweight in older children and adolescents in the United States, Brazil, China, and Russia. Am J Clin Nutr 2002; 75(6):971-917.

2. Cortese S, Falissard B, Angriman M, Pigaiani Y, Banzato C, Bogoni G, Pellegrino M, Cook S, PajnoFerrara F, Bernardina BD, Mouren MC, Maffeis C. The relationship between body size and depression symptoms in adolescents. J Pediatr 2009; 154(1):86-90.

3. Fernandes RA, Zanesco A. Early physical activity promotes lower prevalence of chronic diseases in adulthood. Hypertens Res 2010; 33(9):926-931.

4. Christofaro DG, Chiolero A, Ritti Dias RM, Fernandes RA, Casonatto J, Oliveira AR. Physical activity is inversely associated with high blood pressure independently of overweight in Brazilian adolescents. Scand J Med Sci Sports 2013; 23(3):317-322.

5. Hallal PC, Bertoldi AD, Gonçalves H, Victora CG. Prevalence of sedentary lifestyle and associated factors in adolescents 10 to 12 years of age. Cad Saude Publica 2006; 22(6):1277-1287.

6. Fernandes RA, Junior IF, Cardoso JR, Vaz Ronque ER, Loch MR, de Oliveira AR. Association between regular participation in sports and leisure time behaviors in Brazilian adolescents: a cross-sectional study. BMC 2008; 8:329.

7. Christofaro DG, Fernandes RA, Polito MD, Romanzini M, Ronque ER, Gobbo LA, Oliveira AR. A comparison between overweight cutoff points for detection of high blood pressure in adolescents. $J$ Pediatr (Rio J) 2009; 85(4):353-358.

8. Cole TJ, Flegal KM, Nicholls D, Jackson AA. Body mass index cut offs to define thinness in children and adolescents: international survey. $B M J$ 335(7612):194.

9. Baecke JA, Burema J, Frijters JE. A short questionnaire for the measurement of habitual physical activity in epidemiological studies. Am J Clin Nutr. 1982; 36(5):936-942.

10. Monteiro CA, Benicio MH, Konno SC, Silva AC, Lima AL, Conde WL. Causes for the decline in child under-nutrition in Brazil, 1996-2007. Rev Saude Publica 2009; 43(1):35-43.

11. Ozmen D, Ozmen E, Ergin D, Cetinkaya AC, Sen N, Dundar PE, Taskin EO. The association of self-esteem, depression and body satisfaction with obesity among Turkish adolescents. BMC 2007; 7:80.

12. Branco LM, Hilário MO, Cintra IP. Percepção e satisfação corporal em adolescentes e a relação com seu estado nutricional. Rev Psiq Clin 2006; 33(6):292296.
13. Castro JM, Goldstein SJ. Eating attitudes and behaviors of pre- and post-pubertal females: clues to the etiology of eating disorders. Physiol Behav 1995; 58(1):15-23.

14. Stice E, Shoupak-Neuberg E, Shaw HE, Stein RI. Relation of media exposure to eating disorder symptomatology: an examination of mediating mechanisms. J Abnorm Psychol 1994; 103(4):836-840.

15. Eisenstein E, Ceccon C. Saúde, vida, alegria: manual de educação em saúde com crianças e adolescentes. Rio de Janeiro: CECIP, CEIIAS, ARTMED; 2000.

16. Pinheiro KA, Horta BL, Pinheiro RT, Horta LL, Terres NG, da Silva RA. Common mental disorders in adolescents: a population based cross-sectional study. Rev Bras Psiquiatr 2007; 29(3):241-245.

17. Barros AJ, Hirakata VN. Alternatives for logistic regression in cross-sectional studies: an empirical comparison of models that directly estimate the prevalence ratio. BMC Med Res Methodol 2003; 3:21.

18. Fernandes RA, Conterato I, Messias KP, Christofaro DG, Oliveira AR, Freitas Jr IF. Risk factors associated with overweight among adolescents from western São Paulo State. Rev Esc Enferm USP 2009; 43(4):768-773.

19. Christofaro DG, Andrade SM, Fernandes RA, Ohara D, Dias DF, Freitas Júnior IF, Oliveira DR. Prevalence of risk factors for cardiovascular diseases among students of Londrina - PR: differences between economic classes. Rev Bras Epidemiol 2011; 14(1):27-35.

20. Fernandes RA, Christofaro DG, Cardoso JR, Ronque ER, Freitas Júnior IF, Kawaguti SS, Moraes AC, Oliveira AR. Socioeconomic status as determinant of risk factors for overweight in adolescents. Cien Saude Colet 2011; 16(10):4051-4057.

21. Sales-Peres SH, Goya S, Sant'Anna RM, Silva HM, Sales-Peres AC, Silva RP, Lauris JR, Bastos JR. Prevalence of overweight and obesity, and associated factors in adolescents, at the central west area of the state São Paulo (SP, Brazil). Cien Saude Colet 2010;15(Supl. 2):3175-3184.

22. Costa AS, Chein MB, Tonial SR, Gama ME, Martins ML, Cunha CL, Silva DS, Cruz PR, Brito LM. Nutritional status of adolescents in the state of Maranhão, Brazil, assessed by national and international criteria. Cien Saude Colet 2013; 18(12):3715-3720.

Artigo apresentado em 08/03/2013

Aprovado em 16/05/2013

Versão final apresentada em 21/05/2013 